\title{
Uso de parámetros indirectos para la evaluación de la contaminación de suelos por metales pesados en una zona minera de San Luis Potosí, México
}

\author{
Isabel Pérez-Martínez ${ }^{1}$, Francisco Martín Romero ${ }^{2, *}$ \\ ${ }^{1}$ Posgrado en Ciencias de la Tierra, Universidad Autónoma de México, Ciudad Universitaria, Del. Coyoacán 04510, México, D. F. \\ ${ }^{2}$ Instituto de Geología, Universidad Nacional Autónoma de México, Ciudad Universitaria, Del. Coyoacán 04510, México, D.F. \\ *fmrch@geologia.unam.mx
}

\section{Resumen}

Los residuos mineros son una fuente importante de arsénico y metales pesados que, al estar sujetos a la dispersión eólica e hídrica, pueden ser la causa de la contaminación de suelos en grandes superficies de terreno. En este trabajo, se seleccionó una zona minera en el estado de San Luis Potosí, México, para evaluar el uso de la susceptibilidad magnética (SM), la conductividad eléctrica (CE) y el pH como parámetros indirectos para la delimitación de la distribución espacial de arsénico y metales pesados en los residuos mineros y suelos contaminados por su dispersión. La SM, CE, pH y la concentración total de elementos potencialmente tóxicos (EPT) como arsénico $(\mathrm{As})$, cadmio $(\mathrm{Cd})$, cobre $(\mathrm{Cu})$, plomo $(\mathrm{Pb})$, zinc $(\mathrm{Zn})$ y hierro $(\mathrm{Fe})$ fueron determinados en 107 muestras de residuos y suelos. Además, se analizó la concentración de iones mayores en lixiviados acuosos y se realizaron análisis de difracción de rayos $\mathrm{X}$ y microscopía electrónica de barrido en suelos y jales. Por último, se valoró el índice de peligro (IP) debido a la presencia simultánea de As, Cd y Pb. Los resultados indican que los valores más altos de SM, CE y concentraciones totales de EPT, así como los valores de pH más bajos, se determinaron en los residuos mineros lo cual confirma que son fuente potencial de contaminación de los suelos adyacentes. Por otro lado, indican que en los suelos colindantes con los residuos se determinaron valores altos de estos parámetros. Se demostró que existe una mayor correlación entre la SM, pH y la concentración total de los EPT, y menor para la CE, la cual está determinada por la presencia de sulfatos de calcio (yeso) que es el principal mineral secundario de los procesos de oxidación de los residuos mineros. Se encontró que la relación entre la SM y los EPT está determinada por las fases sólidas (minerales secundarios de Fe) en las que están retenidos los EPT y que presentan una buena respuesta magnética. El análisis de regresión lineal para el índice de peligro (IP) y SM, CE y pH indica una correlación lineal significativa entre IP y SM (0.82), y entre IP y pH (- 0.81); sin embargo, se encontró una correlación lineal más débil entre IP y CE $(\mathrm{r}=0.57)$. Estos resultados permiten concluir que la $\mathrm{CE}$ ayuda a delimitar la fuente de contaminación y la dispersión de los residuos y que la SM y el pH son útiles en la delimitación de la contaminación del suelo, así como en la valoración del grado de contaminación por residuos mineros en la zona de estudio.

Palabras clave: susceptibilidad magnética, conductividad eléctrica, $\mathrm{pH}$, jales mineros, índice de peligro.

\begin{abstract}
Tailings are a significant source of arsenic and heavy metals. When mining wastes are subjected to wind dispersal and water erosion, they may be the cause of widespread soil contamination. In this work, a mining area in the state of San Luis Potosi, Mexico, was selected to evaluate the use of magnetic susceptibility (MS), electrical conductivity (EC) and pH as alternative indicators for the delimitation of the spatial distribution of arsenic and heavy metals in mine wastes and soils contaminated by their dispersal. The MS, $E C, p H$ and the total concentration of potentially toxic elements (PTE), such as arsenic (As), cadmium (Cd), copper (Cu), lead (Pb), zinc $(\mathrm{Zn})$ and iron $(\mathrm{Fe})$, were determined in 107 waste and soil samples. Moreover, the concentration of major ions in solution was analyzed and XRD and SEM analyses were carried out. Finally the hazard index (HI) was assessed as a value showing the effect of joint presence of $\mathrm{As}, \mathrm{Cd}$ and $\mathrm{Pb}$ in the area. The results indicate that higher values of MS, EC and total concentrations of PTE and
\end{abstract}


the lowest $\mathrm{pH}$ values were determined in mining waste, confirming that they are a potential source of soil contamination of adjacent areas. Moreover, soils closest to residues yielded high values for these parameters. There was a strong linear correlation between the MS, $p H$ and the total concentration of PTE and a lower correlation for the EC, which is determined by the presence of calcium sulfate (gypsum) as the main secondary mineral of oxidation processes in mining wastes. Also, the relationship between MS and PTE was determined by solid phases that were contained in the PTE (Fe minerals) with good magnetic response. The linear regression analysis for the hazard index (HI), MS, EC and pH indicates a significant linear correlation between HI and MS (0.82), and between $H I$ and $\mathrm{pH}(-0.81)$. However, only a weak linear correlation between HI and EC was found $(r=0.57)$. These results suggest that the EC will help to determine the source of contamination and the dispersion of the waste and the MS and pH help in the delineation of soil contamination as well as the assessment of the degree of contamination by mining waste in the study area.

Keywords: magnetic susceptibility, electrical conductivity, $\mathrm{pH}$, mine tailings, hazard index.

\section{Introducción}

Los procesos mineros utilizados para la obtención de minerales con valor comercial, generan residuos que constituyen una de las principales causas de la contaminación de los suelos con elementos potencialmente tóxicos (EPT) (Selim y Sparks, 2001). En diferentes regiones de México, las actividades mineras se caracterizan por la generación de grandes cantidades de residuos que contienen EPT que se pueden dispersar al entorno con la subsecuente afectación de los suelos y sedimentos (Gutiérrez-Ruiz et al., 2007; Ongley et al., 2007), donde se acumulan por tiempo indefinido (Fergusson, 1990; Bradl, 2005; Berkowitz et al., 2008), lo que puede representar un riesgo a la salud humana y al ambiente en general.

En las zonas mineras donde el clima predominante es árido o semiárido, el proceso de contaminación de los suelos se da principalmente por la dispersión eólica de los residuos hacia el entorno; mientras que en climas húmedos se da por dispersión hídrica. En ambos casos, ha sido documentado que la dispersión de los residuos mineros afecta grandes extensiones de terreno (Razo et al., 2003; Sultan, 2007; Romero et al., 2008; Navarro et al., 2008).

Para realizar una adecuada evaluación ambiental de la grandes extensiones afectadas por la industria minera se requiere determinar la distribución espacial de EPT, para lo cual es necesario realizar estudios geoquímicos que demandan mucho tiempo, son laboriosos y económicamente caros, en términos de toma, preparación y análisis químico de las muestras; por lo que algún método indirecto puede ser útil en la zonificación del sitio con diferente grado de contaminación, que sirva como guía objetiva para la realización de estudios geoquímicos convencionales en áreas específicas más pequeñas, con lo cual se reduce la cantidad de muestras para la caracterización química y asegurar así, la calidad del estudio de valoración ambiental en estas zonas.

Lo anteriormente expuesto ha motivado la realización del presente trabajo para explorar e investigar los métodos indirectos que pueden ser utilizados para la caracterización de las grandes extensiones de los sitios contaminados por las actividades mineras, considerando la naturaleza química, mineralógica y física de los residuos mineros y de los suelos contaminados por su dispersión.

Algunos autores han reportado que los residuos, que genera la industria minera, contienen una cantidad variable de minerales magnéticos y conductores de electricidad, principalmente asociados a los sulfuros metálicos y óxidos de hierro (Blowes, 1997; Campbell y Fitterman, 2000) los cuales contienen a los metales y metaloides como As, $\mathrm{Cd}$, $\mathrm{Pb}, \mathrm{Cu}, \mathrm{Zn}$, etc., (Holmström y Öhlander, 2001; Levy et al., 1997; Lin, 1997; Romero et al., 2007, 2008; Bea et al., 2010) y cuya dispersión representa un peligro ambiental para los suelos del entorno. También se ha reportado que la generación de drenaje ácido, en este tipo de residuos, es la causa de que sus lixiviados acuosos se caractericen por valores bajos de $\mathrm{pH}$ y altas concentraciones de iones disueltos como $\mathrm{SO}_{4}^{2-} \mathrm{Fe}^{2+}, \mathrm{Fe}^{3+}$ y $\mathrm{Ca}^{2+}$, lo que deriva en altos valores de conductividad eléctrica en estos residuos (Campbell y Fitterman, 2000; Romero et al., 2007), implicando que los suelos afectados por el drenaje ácido de minas pueden acidificarse y aumentar su capacidad de conductividad eléctrica.

Por lo tanto se puede plantear la hipótesis de que la medición de la susceptibilidad magnética, conductividad eléctrica y el $\mathrm{pH}$, en los residuos mineros y en los suelos afectados por su dispersión, puede ser utilizada como una medida indirecta para la identificación de estos residuos y en la delimitación de los suelos afectados por su dispersión.

Aunque ha sido ampliamente demostrado que la medición de la susceptibilidad magnética en los suelos contaminados es eficiente para evaluar la distribución espacial de los metales pesados y por lo tanto para delimitar las zonas con diferente grado de contaminación en áreas industriales urbanas (Fialová et al., 2006; Lu y Bai, 2006; Morton-Bermea et al., 2008), su uso en zonas mineras no ha sido reportada. Así mismo, de acuerdo a nuestra revisión bibliográfica, la conductividad eléctrica y el $\mathrm{pH}$ no han sido utilizados como indicadores indirectos de la contaminación del suelo en zonas mineras.

La realización de la presente investigación se llevó a cabo en una zona minera del estado de San Luís Potosí, en el centro de México, donde estudios anteriores indican que los residuos generados se caracterizan por las relativamente 
altas concentraciones de $\mathrm{As}, \mathrm{Cd}, \mathrm{Pb}, \mathrm{Cu}, \mathrm{Fe}$ y $\mathrm{Zn}$; y aunque no son peligrosos, de acuerdo a los criterios de la normatividad ambiental mexicana (Romero y Gutiérrez, 2010), su dispersión puede haber afectado los suelos del entorno. El objetivo de la investigación es evaluar el uso combinado de la susceptibilidad magnética, conductividad eléctrica y $\mathrm{pH}$ como ayuda en la identificación de residuos mineros y en la delimitación de suelos contaminados dentro del área de influencia debido a la dispersión de residuos y los elementos tóxicos contenidos en ellos.

\section{Materiales y métodos}

\subsection{Descripción del sitio de estudio}

El sitio de estudio se localiza en la parte central de la República Mexicana en el estado de San Luis Potosí (Figura 1). Los climas reportados en la zona, donde se ubica el sitio de estudio, son seco-templado, semiseco-templado y seco-semicálido con valores de temperatura promedio anual igual a $16.9^{\circ} \mathrm{C}$, precipitación pluvial de $391.2 \mathrm{~mm}$ y evaporación potencial de $1942 \mathrm{~mm}$. La dirección de vientos predominantes es hacia el NE y SE (CNA, 2002). Los suelos predominantes en el área son de tipo Litosol eútrico (Ie) y Fluvisol calcárico $(\mathrm{Jk})$ poco desarrollados (CETENAL, 1978). En esta zona se llevan a cabo los procesos de extracción y concentración de minerales económicos de un yacimiento mineral de sulfuros de $\mathrm{Zn}$ $\mathrm{Pb}-\mathrm{Cu}$, del tipo skarn, que ha sido explotado por más de 100 años. La mineralización en el área está constituida por los minerales de mena: esfalerita, galena argentífera, calcopirita, bornita, covelita, malaquita, smithsonita y tetraedrita; y minerales de ganga como pirita, arsenopirita, calcita, danburita y cuarzo. El proceso de beneficio (por flotación selectiva) de los minerales con valor comercial, generó residuos de granulometría fina denominados jales que se han almacenado en un depósito que ocupa una superficie de aproximadamente 95 hectáreas, en el cual se pueden distinguir dos zonas: i) la zona de jales de color gris de reciente depositación, en la parte oeste y ii) la zona de jales inactivos y antiguos de color café-amarillo y rojos en la parte este y sur, respectivamente (Figura 1).

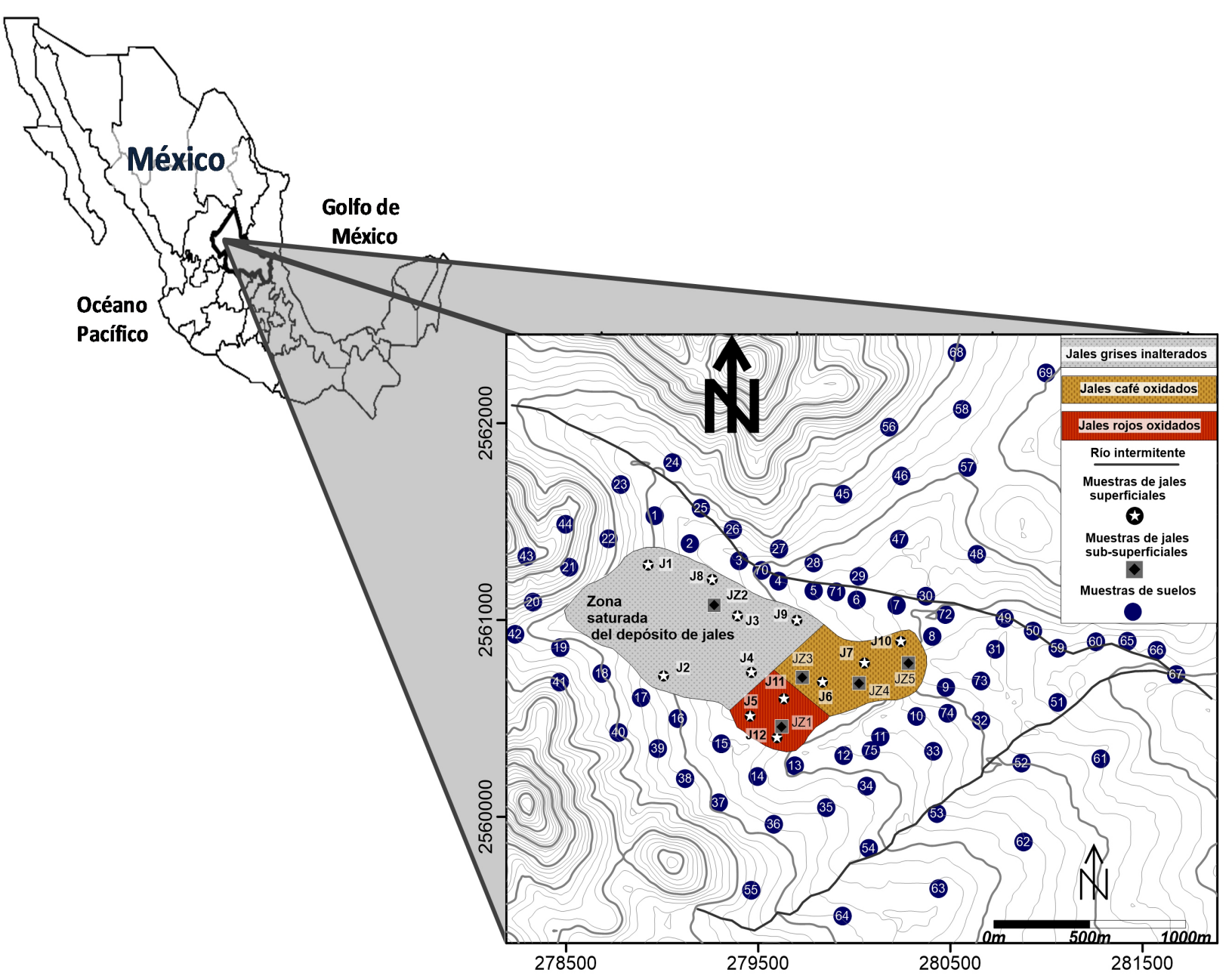

Figura 1. Mapa de ubicación de la zona de muestreo 


\subsection{Muestreo}

Se colectaron un total de 32 muestras de jales (fuente de contaminación) que se distribuyeron en el depósito según su color: 18 muestras de jales de color gris y 14 muestras de jales color café y rojo. De estas muestras, 16 corresponden a jales superficiales y las otra 16 a jales sub-superficiales que se tomaron hasta una profundidad de $1.0 \mathrm{~m}$. Algunas de estas muestras fueron tomadas en campañas de muestreo anteriores y que fueron reportadas por Romero y Gutiérrez (2010). Así mismo, se tomaron 75 muestras de suelos superficiales que se colectaron en la zona donde se presume que éstos presentan diferente grado de contaminación debido a la dispersión de los jales.

\subsection{Análisis de Laboratorio}

Las muestras fueron secadas a temperatura ambiente, disgregadas, homogenizadas y tamizadas (malla de $2 \mathrm{~mm}$ de diámetro). En todas las muestras se determinó conductividad eléctrica $(\mathrm{CE}), \mathrm{pH}$, susceptibilidad magnética específica (SM) y concentración total de arsénico (As), cadmio (Cd), cobre $(\mathrm{Cu})$, hierro $(\mathrm{Fe})$, plomo $(\mathrm{Pb})$ y zinc $(\mathrm{Zn})$. En muestras seleccionadas de jales se obtuvieron lixiviados sintéticos de acuerdo al método descrito por la ASTM (1985) y en los extractos se analizaron los iones mayores en solución $\left(\mathrm{Na}^{+}\right.$, $\mathrm{K}^{+}, \mathrm{Ca}^{2+}, \mathrm{Mg}^{2+}, \mathrm{F}^{-}, \mathrm{Cl}^{-}, \mathrm{NO}_{3}-\mathrm{SO}_{4}{ }^{2-}$ y $\left.\mathrm{HCO}_{3}^{-}\right)$. Con el fin de identificar las fases sólidas a las que están asociados los EPT de interés, algunas muestras de jales y suelos fueron analizadas por difracción de rayos X (DRX) y microscopio electrónico de barrido acoplado con espectrometría de rayos $\mathrm{X}$ por energía dispersa (MEB-EDS).

Para determinar la CE y el $\mathrm{pH}$ se prepararon suspensiones de las muestras homogenizadas en una relación sólido-agua de 1:5. Para determinar la CE, las suspensiones se agitaron durante 1 hora, de acuerdo al método descrito en SSSA Book Series No. 5 parte III (Spark et al., 1996), y para las lecturas se utilizó un conductímetro CORNING 441. Para determinar el $\mathrm{pH}$, las suspensiones fueron agitadas durante 15 minutos, de acuerdo al método $9045 \mathrm{C}$ de la US-EPA (1995) y para las lecturas se utilizó un potenciómetro BECKMAN modelo $\Phi 720$.

En las muestras sólidas homogenizadas se midió la susceptibilidad magnética (SM) utilizando un equipo Bartington MS2 con un sensor de laboratorio de frecuencia dual.

En todas las muestras de jales y suelos se determinó la concentración total de los EPT de interés (As, $\mathrm{Cd}, \mathrm{Cu}, \mathrm{Pb}, \mathrm{Zn}$ y Fe). En las muestras de jales, la concentración total de los EPT de interés se determinó por Espectrometría de Emisión Atómica Inductivamente Acoplada a Plasma (ICP-AES, utilizando un equipo NITTON XLt3 de Thermo Scientific, de acuerdo al método estandarizado 6200 de la US-EPA (2007), que garantiza la determinación del contenido "total" de contaminantes en matrices sólidas sin requerir previamente la digestión ácida en horno de microondas, que sí es requerida por la técnica de Espectrometría de Emisión con Plasma Acoplado Inductivamente (ICP-AES), utilizada en la caracterización realizada en estos mismos jales, por Romero y Gutiérrez (2010). Al respecto es importante señalar que los métodos de digestión de muestras sólidas comúnmente utilizado paras su posterior análisis para su posterior análisis por ICP-AES son los métodos 3051 y 3050B de la US-EPA (1994 y 1996a). En los documentos de la US-EPA sobre estos métodos de digestión se aclara que no garantizan la digestión total para la mayoría de las muestras y que son métodos de digestión ácidos muy agresivos, pero solamente permiten la digestión de los elementos ambientalmente disponibles.

En las muestras de suelos también se determinaron las concentraciones totales de EPT por FRX con el equipo NITTON XLt3. Sin embargo, en algunas muestras de suelo donde las concentraciones totales fueron inferiores al límite de detección de esta técnica, se determinaron por ICP-AES de acuerdo al método estandarizado $6010 \mathrm{~b}$ de la US-EPA (1996b). A pesar de la limitación de este método, descrita anteriormente, para determinar la concentración total, se decidió utilizarlo debido a la necesidad de cuantificar la concentración de EPT en la mayor cantidad posible de muestras para el análisis de regresión con los parámetros indirectos de SM, CE y pH; con el fin de alcanzar los objetivos de esta investigación.

Para valorar la exactitud de los análisis se determinó la concentración de los elementos estudiados en muestras internacionales certificadas: 2710a Montana Soil, Till 4 y RTS 3. Los cálculos indican una exactitud de la técnica de análisis que varía entre 80 y $100 \%$, lo cual muestra un grado de exactitud analíticamente aceptable. Para valorar la precisión de los análisis, al $25 \%$ de las muestras se les determinó la concentración total de EPT por quintuplicado y se calculó el Coeficiente de Variación que varió entre 1 y $12 \%$, que es analíticamente aceptable.

En los análisis de DRX se utilizó un equipo Shimadzu XRD 6000 y en los análisis de MEB-EDS ambiental se utilizó un equipo Jeol 5900-LV para la determinación de las fases sólidas en suelos y jales.

\section{Resultados y discusión}

\subsection{Depósito de Jales: fuente de contaminación}

En el depósito de jales se identificaron, jales visiblemente inalterados de color gris y jales con diferente grado de oxidación de color café y rojo. En la composición mineralógica de los jales de estudio se identificaron minerales primarios y secundarios (Tabla 1), lo que indica diferente grado de alteración. En los jales grises predominan minerales primarios como cuarzo $\left(\mathrm{SiO}_{2}\right)$, calcita $\left(\mathrm{CaCO}_{3}\right)$, pirita $\left(\mathrm{FeS}_{2}\right)$, esfalerita: $(\mathrm{ZnS})$, galena $(\mathrm{PbS})$, arsenopirita (FeAsS) y feldespato potásico $\left(\mathrm{KAlSi}_{3} \mathrm{O}_{8}\right.$ ); pero también se identificaron algunos picos de yeso $\left(\mathrm{CaSO}_{4} \cdot 2 \mathrm{H}_{2} \mathrm{O}\right)$; que 
Tabla 1. Composición mineralógica de las muestras de la zona minera de estudio.

\begin{tabular}{|c|c|}
\hline Minerales primarios & Minerales secundarios \\
\hline \multicolumn{2}{|l|}{ Jales visiblemente inalterados de color gris $(\mathrm{n}=3)$} \\
\hline Cuarzo: $\mathrm{SiO}_{2}$, Calcita: $\mathrm{CaCO}_{3}$ & Yeso: $\mathrm{CaSO}_{4} \cdot 2 \mathrm{H}_{2} \mathrm{O}$ \\
\hline \multicolumn{2}{|l|}{ Pirita: FeS $_{2}$, Esfalerita: $\mathrm{ZnS}$} \\
\hline \multicolumn{2}{|l|}{ Galena: PbS, Arsenopirita: FeAsS } \\
\hline \multicolumn{2}{|l|}{ Fedespato potásico: $\mathrm{KAISi}_{3} \mathrm{O}_{8}$} \\
\hline \multicolumn{2}{|l|}{ Jales oxidados de color café $(n=3)$} \\
\hline Cuarzo: $\mathrm{SiO}_{2}$, & Yeso: $\mathrm{CaSO}_{4} \cdot 2 \mathrm{H}_{2} \mathrm{O}$ \\
\hline Calcita: $\mathrm{CaCO}_{3}$, Pirita: $\mathrm{FeS}_{2}$, Galena: PbS & Hematita: $\mathrm{Fe}_{2} \mathrm{O}_{3}$ \\
\hline \multicolumn{2}{|l|}{ Jales oxidados de color rojo $(n=2)$} \\
\hline Cuarzo: $\mathrm{SiO}_{2}$ & Yeso: $\mathrm{CaSO}_{4} \cdot 2 \mathrm{H}_{2} \mathrm{O}$ \\
\hline Fedespato potásico: $\mathrm{KAISi}_{3} \mathrm{O}_{8}$ & Jarosita: $\mathrm{KFe}_{3}\left(\mathrm{SO}_{4}\right)_{2}(\mathrm{OH})_{6}$ \\
\hline \multirow[t]{2}{*}{ Wollastonita: $\mathrm{CaSiO}_{3}$, Pirita: $\mathrm{FeS}_{2}$} & Hematita: $\mathrm{Fe}_{2} \mathrm{O}_{3}$ \\
\hline & Celestita: $\mathrm{SrSO}_{4}$ \\
\hline \multicolumn{2}{|c|}{ Suelos impactados por la disersión de los jales $(\mathrm{n}=8)$} \\
\hline Cuarzo: $\mathrm{SiO}_{2}$, Fedespato potásico: $\mathrm{KAlSi}_{3} \mathrm{O}_{8}$ & Melanterita: $\mathrm{FeSO}_{4} \cdot 7 \mathrm{H}_{2} \mathrm{O}$ \\
\hline Plagioclasa: $\mathrm{CaAl}_{2} \mathrm{Si}_{2} \mathrm{O}_{8}$, Calcita: $\mathrm{CaCO}_{3}$ & Jarosita: $\mathrm{KFe}_{3}\left(\mathrm{SO}_{4}\right)_{2}(\mathrm{OH})_{6}$ \\
\hline Filosilicatos de tipo esmectítico. & Yeso: $\mathrm{CaSO}_{4} \cdot 2 \mathrm{H}_{2} \mathrm{O}$ \\
\hline Alunita: $\mathrm{KNaAl}_{3}\left(\mathrm{SO}_{4}\right)_{2}(\mathrm{OH})_{6}$ & Hematita: $\mathrm{Fe}_{2} \mathrm{O}_{3}$ \\
\hline Pirita: $\mathrm{FeS}_{2}$, Esfalerita: $\mathrm{ZnS}$, Galena: $\mathrm{PbS}$ & \\
\hline
\end{tabular}

Nota 1: En negritas las faces predominantes.

Nota 2: En cursivas las faces minoritarias.

es un mineral secundario que podría indicar un incipiente proceso de oxidación.

En los jales oxidados de color rojo y café, además del cuarzo $\left(\mathrm{SiO}_{2}\right)$ y feldespato potásico $\left(\mathrm{KAlSi}_{3} \mathrm{O}_{8}\right)$ que son minerales primarios muy resistentes a la oxidación, se identificaron predominantemente minerales secundarios como yeso $\left(\mathrm{CaSO}_{4} \cdot 2 \mathrm{H}_{2} \mathrm{O}\right)$ y hematita $\left(\mathrm{Fe}_{2} \mathrm{O}_{3}\right)$ que son los minerales más comunes que caracterizan a los jales oxidados (McGregor et al., 1998; Johnson et al., 1999; Romero et al., 2007). Adicionalmente, en los jales oxidados de color rojo se identificaron jarosita $\left(\mathrm{KFe}_{3}\left(\mathrm{SO}_{4}\right)_{2}(\mathrm{OH})_{6}\right)$ y celestita $\left(\mathrm{SrSO}_{4}\right)$. Es importante señalar que en los jales oxidados aún se identificaron algunas fases minoritarias que corresponden a minerales primarios como la calcita $\left(\mathrm{CaCO}_{3}\right)$, wollastonita $\left(\mathrm{CaSiO}_{3}\right)$ y algunos sulfuros metálicos como la pirita $\left(\mathrm{FeS}_{2}\right)$, esfalerita $(\mathrm{ZnS})$ y galena $(\mathrm{PbS})$.

Por otro lado, los resultados de los análisis de $\mathrm{pH}$, conductividad eléctrica (CE), susceptibilidad magnética (SM) y concentración total de elementos potencialmente tóxicos (EPT) en ambos jales se presentan en la Tabla 2.

En los jales inalterados de color gris los valores de $\mathrm{pH}$ varían entre 6.5 y 9.3 con un valor promedio de 7.7 ; que indica condiciones neutras y básicas, lo que significa que actualmente no son generadores de drenaje ácido. Así mismo, la CE en estos jales inalterados varía entre 502 y $2130 \mu \mathrm{S} / \mathrm{cm}$ con una media de $1550 \mu \mathrm{S} / \mathrm{cm}$. Valores relativamente altos de $\mathrm{CE}$ en estos jales inalterados pueden ser explicados por la presencia de los sulfuros metálicos como la pirita, esfalerita y galena, los cuales han sido reportados como minerales conductores de electricidad (Campbell y Fitterman, 2000) o por sales solubles residuales que son utilizadas como depresores en el proceso de flotación selectiva, que han generado los jales de estudio, tales como los sulfatos de $\mathrm{Zn}$ o de $\mathrm{Cu}$ (Gutiérrez-Ruiz et al., 2007).

Por otro lado, los valores de $\mathrm{pH}$ de los jales oxidados de color café varían entre 6.6 y 8.2 con una media de 7.6, y los valores de la CE varían entre 1384 y $2320 \mu \mathrm{S} / \mathrm{cm}$ con una media de $1939 \mu \mathrm{S} / \mathrm{cm}$; mientras que en los jales oxidados de color rojo el $\mathrm{pH}$ varía entre 4.1 y 8.4 con una media de 5.9 , y la CE entre 2200 y $2590 \mu \mathrm{S} / \mathrm{cm}$ con una media de $2327 \mu \mathrm{S} / \mathrm{cm}$. Estos valores de $\mathrm{pH}$ indican condiciones desde medianamente ácidas hasta neutras y básicas, lo que sugiere que la acidez generada, por la oxidación de los sulfuros metálicos (Ecuación 1), fue neutralizada por la disolución de minerales alcalinos, principalmente calcita y wollastonita (Ecuación 2 y 3 ), con mayor eficiencia en los jales de color café que en los de color rojo.

La liberación de sulfatos $\left(\mathrm{SO}_{4}^{2-}\right)$, generados por la oxidación de los sulfuros metálicos, principalmente pirita (Ecuación 1), junto con la liberación de algunos cationes como el $\mathrm{Ca}^{2+}$, debido a la disolución, principalmente de la calcita y wollastonita, durante el proceso de neutralización (Ecuación 2 y 3) puede explicar los altos valores de conductividad eléctrica determinados en los jales oxidados de color café y rojo, que son mayores que los determinados 
en los jales grises inalterados.

$$
\begin{array}{ll}
\mathrm{FeS}_{2}+7 / 2 \mathrm{O}_{2}+\mathrm{H}_{2} \mathrm{O} \leftrightarrow \mathrm{Fe}^{2+}+2 \mathrm{SO}_{4}{ }^{2-}+2 \mathrm{H}^{+} & \text {Ecuación 1 } \\
\mathrm{CaCO}_{3}+\mathrm{H}^{+} \leftrightarrow \mathrm{Ca}^{2+}+\mathrm{HCO}_{3}^{-} & \text {Ecuación 2 } \\
\mathrm{CaSiO}_{3}+\mathrm{H}_{2} \mathrm{O}+2 \mathrm{H}^{+} \rightarrow \mathrm{Ca}^{2+}+\mathrm{H}_{4} \mathrm{SiO}_{4} & \text { Ecuación 3 }
\end{array}
$$

Bajo las condiciones medianamente ácidas hasta neutras y básicas, observadas en los jales oxidados, se favorece la precipitación de minerales secundarios de hierro como lo demuestra la presencia de hematita y jarosita que fueron debidamente identificados por Difracción de Rayos X (Tabla 1).

La susceptibilidad magnética (SM) en los jales de estudio varía en un amplio intervalo $(\mathrm{SM}=7.7$ - $1393.7 \mathrm{x}$ $10^{-8} \mathrm{~m}^{3} / \mathrm{kg}$ ). En los jales inalterados de color gris, los valores de la SM varían entre 7.7 y $36.1 \times 10^{-8} \mathrm{~m}^{3} / \mathrm{kg}$ con un valor promedio de $23.8 \times 10^{-8} \mathrm{~m}^{3} / \mathrm{kg}$; que son valores bajos con respecto a los determinados en los en los jales oxidados de color café que varían entre 37.9 y $87.2 \times 10^{-8} \mathrm{~m}^{3} / \mathrm{kg}$, con un valor promedio de $64.4 \times 10^{-8} \mathrm{~m}^{3} / \mathrm{kg}$. Finalmente, en los jales oxidados rojos se determinaron los valores más altos de este parámetro físico que varía entre 497.8 y 1393.7 x $10^{-8} \mathrm{~m}^{3} / \mathrm{kg}$, con un valor promedio de $974.2 \times 10^{-8} \mathrm{~m}^{3} / \mathrm{kg}$.

De acuerdo a los datos de SM, los valores de este parámetro, en los jales inalterados grises de este estudio, pueden corresponder a minerales clasificados como paramagnéticos como la pirita (Dearing, 1994); cuya presencia ha sido demostrada en estos jales. En el caso de los jales oxidados de color café y rojo, los valores de SM pueden corresponder a hematita que es el mineral secundario de hierro identificado en estos jales oxidados y que ha sido clasificado como antiferromagnético (Dearing, 1994). Los valores más altos de SM en los jales rojos podrían corresponder a la jarosita identificada en estos jales y que es clasificada como un mineral paramagnético con un acoplamiento antiferromagnético de los iones férricos (López-Andreo et al., 1987).

Las concentraciones totales de los elementos potencialmente tóxicos (EPT) analizados, también varían en un amplio intervalo. En términos de valores promedio,

\begin{tabular}{|c|c|c|c|c|c|c|c|c|c|}
\hline Estadígrafo & pH & $\begin{array}{c}\mathbf{C E} \\
(\mu \mathrm{S} / \mathrm{cm})\end{array}$ & $\begin{array}{c}\mathrm{SM} \\
\left(10^{-8} \mathrm{~m}^{3} / \mathrm{kg}\right)\end{array}$ & As & Cd & $\begin{array}{c}\mathrm{Cu} \\
(\mathrm{mg} / \mathrm{kg})\end{array}$ & $\mathbf{P b}$ & $\mathbf{Z n}$ & $\begin{array}{l}\mathrm{Fe} \\
(\%)\end{array}$ \\
\hline \multicolumn{10}{|c|}{ Jales inalterados de color gris $(n=18)$} \\
\hline Mínimo & 6.5 & 502 & 7.7 & 135 & 25 & 213 & 190 & 1466 & 2.36 \\
\hline Máximo & 9.3 & 2130 & 36.1 & 427 & 434 & 6597 & 10947 & 38569 & 5.16 \\
\hline Media & 7.7 & 1550 & 23.8 & 261 & 86 & 904 & 1411 & 7360 & 3.86 \\
\hline Desv. Est. & 0.6 & 478 & 9.1 & 79 & 93 & 1455 & 2468 & 8542 & 0.73 \\
\hline \multicolumn{10}{|c|}{ Jales oxidados de color café $(n=9)$} \\
\hline Mínimo & 6.6 & 1384 & 37.9 & 201 & 34 & 190 & 210 & 2280 & 2.54 \\
\hline Máximo & 8.2 & 2320 & 87 & 834 & 320 & 1086 & 2280 & 13775 & 4.69 \\
\hline Media & 7.6 & 1939 & 64.4 & 323 & 108 & 552 & 1046 & 7028 & 3.37 \\
\hline Desv. Est. & 0.5 & 341 & 17.4 & 198 & 91 & 325 & 701 & 3903 & 0.72 \\
\hline \multicolumn{10}{|c|}{ Jales oxidados de color rojo $(n=5)$} \\
\hline Mínimo & 4.1 & 2200 & 497.8 & 1074 & 690 & 2970 & 10670 & 29940 & 8.39 \\
\hline Máximo & 8.4 & 2590 & 1393.7 & 1893 & 2479 & 9804 & 60000 & 128611 & 24.9 \\
\hline Media & 5.9 & 2327 & 974.2 & 1472 & 1848 & 7361 & 38078 & 85819 & 17.49 \\
\hline Desv. Est. & 1.7 & 162 & 354.8 & 301 & 678 & 2592 & 19268 & 35967 & 6.12 \\
\hline \multicolumn{10}{|c|}{ Suelos afectados por la dispersión de los jales } \\
\hline Mínimo & 7.2 & 67 & 23.7 & BLD & BLD & BLD & 20 & 90 & 0.89 \\
\hline Máximo & 9 & 2510 & 275.4 & 1773 & 2440 & 4030 & 9250 & 72880 & 6.47 \\
\hline Media & 8.2 & 337 & 83.8 & 133 & 95 & 334 & 791 & 5201 & 2.19 \\
\hline Desv. Est. & 0.4 & 565 & 43.6 & 233 & 317 & 687 & 1476 & 11963 & 0.82 \\
\hline
\end{tabular}
las concentraciones totales de EPT son mayores en los jales oxidados rojos, comparadas con las de los jales oxidados

Tabla 2. Estadística descriptiva de los parámetros indirectos $(\mathrm{pH}, \mathrm{CE}$ y SM) y de las concentraciones totales de los elementos potencialmente tóxicos analizados.

$\mathrm{BLD}=$ Bajo el límite de detección.

Los límites de detección para de la técnica de FRX (mg/kg) son: $\mathrm{As}=14, \mathrm{Cd}=20, \mathrm{Cu}=43, \mathrm{~Pb}=4, \mathrm{Zn}=61$ y Fe $=114$.

Los límites de detección de la técnica de ICP-AES $(\mathrm{mg} / \mathrm{kg})$ son: $\mathrm{As}=4, \mathrm{Cd}=6, \mathrm{Cu}:=3.5, \mathrm{~Pb}=1.0, \mathrm{Zn}=5$ y Fe $=10$. 
café y los jales inalterados grises.

Las mayores concentraciones de EPT corresponden a los jales oxidados de color rojo y café, donde se determinaron los mayores valores de SM y CE y que se caracterizan por la presencia de minerales secundarios de hierro como hematita y jarosita. Por otro lado, los resultados también indican que en los jales grises inalterados las concentraciones totales de EPT son relativamente altas y corresponden con los valores más bajos de SM y relativamente altos de $\mathrm{CE}$, lo que puede deberse a los sulfuros metálicos que predominan en estos jales; que además de ser la fuente primaria de los EPT como $\mathrm{As}, \mathrm{Cd}, \mathrm{Cu}, \mathrm{Pb}, \mathrm{Fe}$ y $\mathrm{Zn}$ son minerales que pueden ser clasificados como paramagnéticos (Dearing, 1994) y buenos conductores de electricidad (Blowes, 1997; Campbell y Fitterman, 2000).

3.2. Suelos afectados por la dispersión de los jales de estudio.

En los suelos afectados, por la dispersión de los jales de estudio, predominan minerales primarios como cuarzo $\left(\mathrm{SiO}_{2}\right)$, feldespato potásico $\left(\mathrm{KAlSi}_{3} \mathrm{O}_{8}\right)$, plagioclasa $\left(\mathrm{CaAl}_{2} \mathrm{Si}_{2} \mathrm{O}_{8}\right)$, calcita $\left(\mathrm{CaCO}_{3}\right)$, filosilicatos de tipo esmectítico y alunita $\left(\mathrm{KNaAl}_{3}\left(\mathrm{SO}_{4}\right)_{2}(\mathrm{OH})_{6}\right)$ (Tabla 1). Además, como una evidencia de que estos suelos están afectados por la dispersión de los jales de estudio, se identificaron algunas fases minoritarias de sulfuros metálicos, asociados a los jales inalterados, como pirita $\left(\mathrm{FeS}_{2}\right)$, esfalerita $(\mathrm{ZnS})$ y galena $(\mathrm{PbS})$, y de minerales secundarios, que se formaron en los jales oxidados, como melanterita $\left(\mathrm{FeSO}_{4} * 7 \mathrm{H}_{2} \mathrm{O}\right)$, jarosita $\left(\mathrm{KFe}_{3}\left(\mathrm{SO}_{4}\right)_{2}(\mathrm{OH})_{6}\right)$, yeso $\left(\mathrm{CaSO}_{4} \cdot 2 \mathrm{H}_{2} \mathrm{O}\right)$ y hematita $\left(\mathrm{Fe}_{2} \mathrm{O}_{3}\right)$.

Los valores de $\mathrm{pH}(7.2-9.0)$, SM (23.7 - $275.4 \mathrm{x}$ $\left.10^{-8} \mathrm{~m}^{3} / \mathrm{kg}\right)$ y $\mathrm{CE}(67-2,510 \mu \mathrm{S} / \mathrm{cm})$ varían en un amplio intervalo. Así mismo, las concentraciones totales de EPT en estos suelos, también varían en un amplio intervalo (Tabla 2).

Esta amplia variación de los parámetros indirectos $(\mathrm{pH}$, CE y SM) y de las concentraciones totales de EPT sugiere diferente grado de contaminación debido a la dispersión de los diferentes tipos de jales del depósito. Los valores más altos de la SM y CE, y de las concentraciones totales de EPT se determinaron en muestras de suelos ubicados alrededor inmediato del depósito de jales a distancias entre 100 y $300 \mathrm{~m}$; aunque también se observaron valores altos de estas variables, en lo suelos contaminados, hasta unos $750 \mathrm{~m}$ hacia el SE que son sitios topográficamente más bajos con relación a la ubicación del depósito de jales y coincidente con la dirección preferencial de vientos reportada.

Es importante señalar la existencia de dos quebradas a unos $1000 \mathrm{~m}$ hacia el SE y a unos $150 \mathrm{~m}$ hacia el $\mathrm{N}$ y NE del depósito de jales (Figura 1), que actúan como barreras físicas que limitan la dispersión de los jales a mayores distancias, a pesar de que los vientos predominantes hacia el SE y NE favorecería la dispersión eólica de estos residuos.
3.3. Análisis de la relación entre $\mathrm{pH}, \mathrm{CE}$ y $\mathrm{SM}$ con las concentraciones totales de los EPT en las muestras de jales y suelos contaminados de la zona de estudio.

En la Tabla 3 se presenta la matriz de correlación entre los parámetros indirectos ( $\mathrm{pH}, \mathrm{CE}$ y $\mathrm{SM})$ y las concentraciones totales de los EPT analizados, tanto en las muestras de jales como en los suelos contaminados de la zona minera.

Coeficientes de correlación entre 0.73 y 0.95 entre las concentraciones totales de los EPT analizados indica una fuerte correlación lineal positiva entre ellos, lo que parece indicar que están asociados a fases sólidas comunes. Una correlación lineal fuerte y positiva entre la SM y las concentraciones totales de $\mathrm{As}, \mathrm{Cd}, \mathrm{Cu}, \mathrm{Pb}, \mathrm{Zn}$ y Fe, se interpreta a partir de los coeficientes de correlación entre estos parámetros que varían de 0.71 a 0.78 . Así mismo, los coeficientes de correlación de 0.81 a 0.95 entre el contenido de Fe y las concentraciones totales de los EPT analizados indican la fuerte correlación lineal entre ellos; lo que permite inferir una fuerte asociación entre $\mathrm{As}, \mathrm{Cd}, \mathrm{Cu}, \mathrm{Pb}$ y $\mathrm{Zn}$ con los minerales de $\mathrm{Fe}$ como por ejemplo la hematita y jarosita, que son minerales secundarios de Fe, identificados es los jales oxidados estudiados (Tabla 1).

Por otro lado, el análisis de los resultados indica que existe una fuerte correlación lineal negativa entre el $\mathrm{pH}$ y las concentraciones totales de EPT con coeficientes de correlación entre -0.63 y -0.80 . Así mismo, los datos presentados en la Tabla 3 indican que existe una correlación lineal negativa entre el $\mathrm{pH}$ y la $\mathrm{CE}(\mathrm{r}=-0.68)$, que se puede explicar por los procesos de oxidación-neutralización que han ocurrido en los jales oxidados de color café y rojos (fuente de contaminación de los suelos), que ha propiciado: i) la disminución del $\mathrm{pH}$, ii) la liberación de iones como el

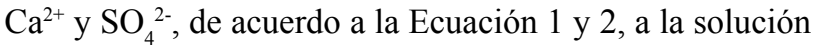
de poro que es la causa del aumento en la conductividad eléctrica y iii) la precipitación de minerales secundarios de $\mathrm{Fe}$, a los que podrían estar asociados los EPT por procesos de sorción, como ha sido reportado por Romero et al. (2010) en este tipo de jales.

De acuerdo a los datos de la Tabla 3 también se observa una correlación lineal positiva entre la CE y las concentraciones totales de EPT, con coeficientes de correlación entre 0.52 y 0.62 . Este comportamiento indica que los EPT están asociados a minerales conductores de electricidad como la pirita y los sulfuros metálicos, que son la fuente de los EPT analizados, en el caso de los jales inalterado grises. En el caso de jales oxidados y suelos afectados por su dispersión, la buena correlación entre CE y los EPT se puede explicar por la presencia de minerales secundarios, a los que están asociados los EPT vía sorción, que bajo las condiciones ambientales pueden liberar iones a la solución de poro que es la causa del aumento de la conductividad eléctrica.

En la Tabla 4 se presentan los resultados del análisis de los lixiviados acuosos obtenidos en muestras de jales para 
Tabla 3. Matriz de correlación entre los parámetros indirectos (SM, CE y $\mathrm{pH}$ ) y las concentraciones totales de los EPT.

\begin{tabular}{lrrrrrrrrr}
\hline & SM & pH & \multicolumn{1}{c}{ CE } & \multicolumn{1}{c}{ As } & Cd & $\mathbf{C u}$ & $\mathbf{F e}$ & Pb & $\mathbf{Z n}$ \\
\hline SM & 1 & -0.48 & 0.34 & 0.77 & 0.78 & 0.75 & 0.76 & 0.73 & 0.71 \\
$\mathbf{p H}$ & & 1 & -0.67 & -0.64 & -0.73 & -0.83 & -0.81 & -0.82 & -0.84 \\
$\mathbf{C E}$ & & & 1 & 0.62 & 0.52 & 0.58 & 0.53 & 0.45 & 0.55 \\
$\mathbf{A s}$ & & & & 1 & 0.73 & 0.75 & 0.82 & 0.73 & 0.75 \\
$\mathbf{C d}$ & & & & & 1 & 0.89 & 0.81 & 0.82 & 0.91 \\
$\mathbf{C u}$ & & & & & & 1 & 0.88 & 0.92 & 0.94 \\
$\mathbf{F e}$ & & & & & & & 1 & 0.95 & 0.88 \\
$\mathbf{P b}$ & & & & & & & & 1 & 0.92 \\
$\mathbf{Z n}$ & & & & & & & & & 1 \\
\hline
\end{tabular}

simular la solución de poro, que indican el predominio de

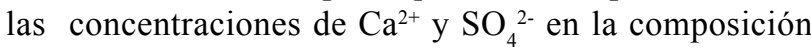
química de los lixiviados y en esa misma tabla se observan las correlaciones entre estos iones y la $\mathrm{CE}$, dando como resultado una fuerte correlación lineal entre la $\mathrm{CE}$ y $\mathrm{Ca}^{2+}$ $(\mathrm{r}=0.74)$ y entre la $\mathrm{CE}$ y SO${ }_{4}{ }^{2-}(\mathrm{r}=0.76)$. Además, entre las concentraciones de $\mathrm{Ca}^{2+} \mathrm{y} \mathrm{SO}_{4}^{2-}$ se encontró una correlación positiva extremadamente alta $(\mathrm{r}=0.99)$, lo que sugiere que la principal fuente de estos iones es la disolución del yeso que es un mineral secundario debidamente identificado en los jales de estudio (Tabla 1).

3.4. Identificación de fases sólidas a las que está asociados los EPT de interés.

El análisis por MEB-EDS permitió identificar partículas en cuya composición sobresale el Fe y los EPT de interés: $\mathrm{As}, \mathrm{Cu}, \mathrm{Pb}$ y $\mathrm{Zn}$ (Figura 2). Se infiere que estas partículas pueden corresponder a los minerales secundarios de hierro que fueron identificados en los jales oxidados por DRX (Tabla 1), que contienen en su estructura al $\mathrm{As}, \mathrm{Cu}, \mathrm{Pb}$ y $\mathrm{Zn}$.

Estos resultados son congruentes con el hecho de que el arsénico y los metales pesados pueden estar asociados a los minerales secundarios de Fe debido a procesos de sorción; tal como ha sido ampliamente reportado en la literatura (Goldberg, 1986; Fuller et al., 1993; Bowell, 1994; Lin, 1997; Foster et al., 1998; McGregor et al., 1998; Romero et al., 2007).

Los resultados de MEB-EDS puede ser la explicación de las altas correlaciones encontradas entre las concentraciones totales de EPT y los valores de susceptibilidad magnética (SM) de los minerales secundarios de Fe (hematita y jarosita) que se clasifican como minerales antiferromagnéticos (Evans y Heller, 2003), que se caracterizan por valores medios y altos de SM.

3.5. Parámetros indirectos ( $\mathrm{SM}, \mathrm{CE}$ y $\mathrm{pH})$ como indicadores del índice de peligro del sitio de estudio.

Para valorar la situación general de peligro ambiental como resultado de la contribución simultánea de los EPT
(As, $\mathrm{Cd} \mathrm{y} \mathrm{Pb}$ ) regulados en la normatividad ambiental mexicana (NOM-147-SEMARNAT/SSA1-2004), se propone calcular el índice de peligro, para estos elementos, utilizando las concentraciones máximas permisibles (CMP) señaladas en la NOM-147: As $=22 \mathrm{mg} / \mathrm{kg}, \mathrm{Cd}=37 \mathrm{mg} / \mathrm{kg}$ y $\mathrm{Pb}=400 \mathrm{mg} / \mathrm{kg}$.

El índice de peligro se calculó a través de la Ecuación 4:

$$
\mathrm{IP}=\left[\left(\mathrm{CT}_{\mathrm{As}} / \mathrm{CMP}_{\mathrm{As}}+\mathrm{CT}_{\mathrm{Cd}} / \mathrm{CMP}_{\mathrm{Cd}}+\mathrm{CT}_{\mathrm{Pb}} / \mathrm{CMP}_{\mathrm{Pb}}\right)\right] / 3
$$

$\mathrm{CT}_{\mathrm{As}}, \mathrm{CT}_{\mathrm{Cd}}, \mathrm{CT}_{\mathrm{Pb}}$ : Concentraciones totales de As, $\mathrm{Cd} \mathrm{y}$ $\mathrm{Pb}$ en las muestras de la zona de estudio.

$\mathrm{CMP}_{\mathrm{As}}, \mathrm{CMP}_{\mathrm{Cd}}, \mathrm{CMP}_{\mathrm{Pb}}$ : Concentraciones Máximas Permisibles señaladas en la NOM-147-SEMARNAT/ SSA1-2004.

Los valores de IP calculados para la zona varían de 0.02 a 91, lo que indica que hay áreas con diferente grado de peligrosidad: Peligro nulo (IP $<1)$, Peligro bajo (IP $=1-3$ ), Peligro alto $(\mathrm{IP}=3-10)$ y Peligro extremo (IP $>10)$. Dada la clasificación anterior, del total de muestras analizadas $(\mathrm{n}=$ 107), en el $28 \%$ la presencia del $\mathrm{As}, \mathrm{Cd}$ y $\mathrm{Pb}$ no representa ningún peligro (IP $<1$ ), en el $21 \%$ el peligro es bajo (IP $=1-3)$, en el $40 \%$ el peligro es alto $(\mathrm{IP}=3-10) \mathrm{y}$ en el $11 \%$ el peligro es extremo (IP $>10)$.

Considerando que el IP aporta información cuantitativa acerca de la problemática ambiental relacionada con los residuos y suelos contaminados, se realizó la matriz de correlación (Tabla 5) entre IP y los parámetros medidos (SM, $\mathrm{CE} \mathrm{y} \mathrm{pH}$ ) con el fin de valorar el grado de dependencia de este con los parámetros y así demostrar la utilidad de éstos últimos como indicadores indirectos de la problemática ambiental de la zona estudiada.

En la Tabla 5 se puede observar que la correlación lineal más significativa ocurre entre la SM y el IP $(0.82)$ y entre el $\mathrm{pH}$ y el IP (-0.81). Una menor correlación lineal se observó entre la CE y el IP (0.57).

En la Figura 3 se muestra el mapa de distribución de los valores de IP asociados a las anomalías de $\mathrm{SM}, \mathrm{CE}$ y $\mathrm{pH}$. De acuerdo a los valores de SM en los suelos se delimitaron cuatro tipos de anomalías: "baja", "intermedia", "alta" y "extrema". La anomalía "baja", corresponde a valores de SM inferiores a valor promedio $(\bar{x})$, determinado en suelos. El valor promedio de la SM se asume como el valor de fondo natural en la zona de estudio. En la zona minera la SM promedio $(\bar{x})$ es de $84 \times 10^{-8} \mathrm{~m}^{3} / \mathrm{kg}$; Las anomalía "intermedia", "alta" y "muy alta" corresponden a valores de $1.5 * \bar{x}, 2 * \bar{x}$ y $5 * \bar{x}$, respectivamente.

Los valores de IP que indican un grado de peligrosidad nulo (IP $<1.0)$ y bajo $(\mathrm{IP}=1-3)$ corresponden a las áreas de anomalías de susceptibilidad magnética "baja" (SM < $84 \times 10^{-8} \mathrm{~m}^{3} / \mathrm{kg}$ ); mientras que los valores que indican un grado de peligrosidad alto (IP $=3-10$ ) y extremo (IP $>10)$ se localizan en las áreas de anomalías de SM "intermedia" $\left(\mathrm{SM}=84-126 \times 10^{-8} \mathrm{~m}^{3} / \mathrm{kg}\right)$, "alta" $\left(\mathrm{SM}=126-336 \times 10^{-8}\right.$ $\left.\mathrm{m}^{3} / \mathrm{kg}\right)$ y "muy alta" $\left(\mathrm{SM}=>336 \times 10^{-8} \mathrm{~m}^{3} / \mathrm{kg}\right)$. 

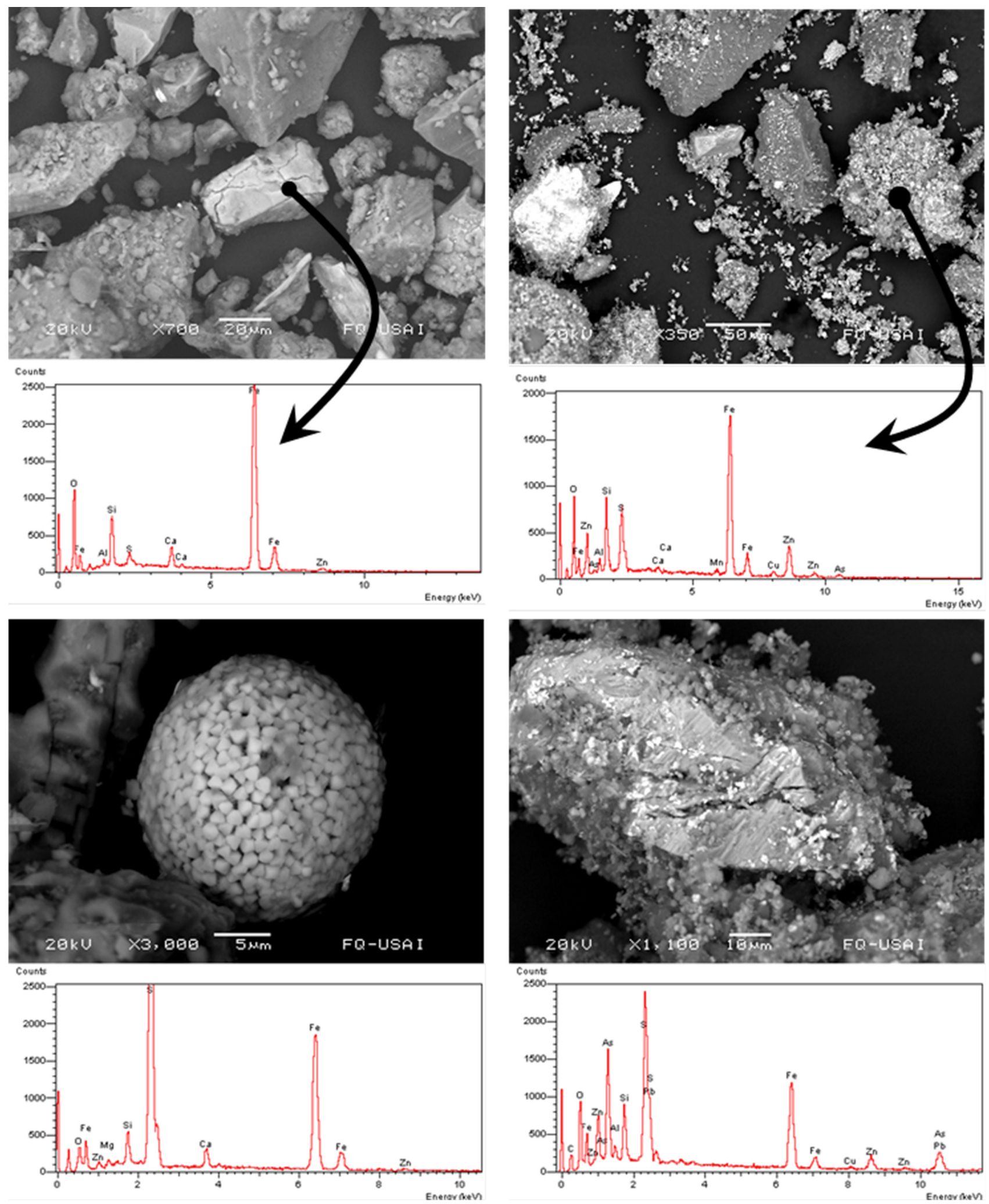

Figura 2. Imágenes de microscopio electrónico de barrido (MEB) y los espectros representativos de los análisis EDS en algunas de las partículas observadas. 
Tabla 4. Concentración de iones mayores en lixiviados acuosos y matriz de correlación entre CE y los iones mayores.

\begin{tabular}{|c|c|c|c|c|c|c|c|c|c|}
\hline Estadígrafo & $\mathrm{Na}^{+}$ & $\overline{\mathbf{K}^{+}}$ & $\mathrm{Ca}^{2+}$ & $\mathrm{Mg}^{2+}$ & $\begin{array}{c}F^{-} \\
(\mathrm{mg} / \mathrm{L})\end{array}$ & $\overline{\mathrm{Cl}^{-}}$ & $\mathrm{NO}_{3}^{-}$ & $\mathrm{SO}_{4}{ }^{2-}$ & $\overline{\mathrm{HCO}_{3}}$ \\
\hline Máximo & 14.51 & 18.78 & 581.5 & 5.54 & 0.59 & 10.91 & 39.96 & 1224 & 114.2 \\
\hline Mínimo & 0.09 & 0.75 & 18.05 & 0.02 & 0.08 & 0.23 & 0.92 & 2.98 & 19.87 \\
\hline Media & 3.44 & 3.89 & 246.5 & 1.63 & 0.29 & 2.53 & 6.91 & 516 & 39.44 \\
\hline Desv. Est. & 3.78 & 5.03 & 220 & 1.49 & 0.13 & 2.49 & 11.62 & 471.4 & 24.88 \\
\hline $\mathrm{Na}^{+}$ & $\mathbf{K}^{+}$ & $\mathrm{Ca}^{2+}$ & $\mathbf{M g}^{2+}$ & $\mathbf{F}^{-}$ & $\mathrm{Cl}^{-}$ & $\mathrm{NO}_{3}^{-}$ & $\mathrm{SO}_{4}{ }^{2-}$ & $\mathrm{HCO}_{3}^{-}$ & $\mathbf{C E}$ \\
\hline $\mathrm{Na}^{+}$ & 0.17 & -0.03 & 0.19 & 0 & 0.38 & 0.59 & -0.05 & 0.12 & 0.02 \\
\hline $\mathbf{K}^{+}$ & 1 & 0.19 & 0.45 & 0.66 & 0.74 & 0.35 & 0.16 & 0.33 & 0.02 \\
\hline $\mathrm{Ca}^{2+}$ & & 1 & 0.52 & 0.36 & 0.25 & -0.22 & 0.99 & -0.55 & 0.74 \\
\hline $\mathrm{Mg}^{2+}$ & & & 1 & 0.24 & 0.68 & -0.33 & 0.54 & -0.49 & 0.38 \\
\hline $\mathbf{F}^{-}$ & & & & 1 & 0.31 & 0.26 & 0.33 & 0.26 & -0.07 \\
\hline $\mathrm{Cl}^{-}$ & & & & & 1 & 0.02 & 0.24 & 0.08 & 0.17 \\
\hline $\mathrm{NO}_{3}^{-}$ & & & & & & 1 & -0.26 & 0.54 & -0.21 \\
\hline $\mathrm{SO}_{4}{ }^{2-}$ & & & & & & & 1 & -0.6 & 0.76 \\
\hline $\mathrm{HCO}_{3}^{-}$ & & & & & & & & 1 & -0.6 \\
\hline $\mathrm{CE}$ & & & & & & & & & 1 \\
\hline
\end{tabular}

Tabla 5. Matriz de correlación del IP \& SM, pH y CE.

\begin{tabular}{|c|c|c|c|c|}
\hline & SM & pH & CE & IP \\
\hline SM & 1 & -0.48 & 0.34 & 0.82 \\
\hline pH & & 1 & -0.67 & -0.81 \\
\hline $\mathbf{C E}$ & & & 1 & 0.57 \\
\hline IP & & & & 1 \\
\hline
\end{tabular}

Sin embargo, se observa que en algunas áreas de anomalías de $\mathrm{SM}$ "baja" (SM $<84 \times 10^{-8} \mathrm{~m}^{3} / \mathrm{kg}$ ) se localizan valores de IP que corresponden a un grado de peligrosidad "alto" (IP = $3-10)$, lo que se puede explicar debido a la presencia de algunos sulfuros metálicos, que han sido reportados en los residuos, que son la fuente primaria de los EPT como $\mathrm{As}, \mathrm{Pb}$ y $\mathrm{Cd}$ y que son minerales que pueden ser clasificados como paramagnéticos (Dearing, 1994).

Por otro lado, se pueden observar áreas hacia el NE del depósito de jales donde las anomalías de la SM son "intermedias" ( $\mathrm{SM}=84-126 \times 10^{-8} \mathrm{~m}^{3} / \mathrm{kg}$ ) pero que los valores del IP son inferiores a 1 (grado de peligro nulo), lo que pudiera indicar que no están afectados por la dispersión de los jales. Este comportamiento parece indicar que en la zona existen minerales magnéticos de origen natural, adicional a los asociados con los jales de estudio.

Para la $\mathrm{CE}$, se observaron áreas con un grado de peligro "alto" (IP = 3-10) y "muy alto" (IP > 10) que corresponden a valores de CE mayor a $506 \mu \mathrm{S} / \mathrm{cm}$, equivalente a 1.5 veces el valor promedio determinado en suelos $(337 \mu \mathrm{S} / \mathrm{cm})$ que se asume como valor de fondo. En las áreas con valores de $\mathrm{CE}$ menor a $337 \mu \mathrm{S} / \mathrm{cm}$, se asocian valores de IP $<1$ (peligro nulo); y en las áreas con CE entre 337 y $506 \mu \mathrm{S} / \mathrm{cm}$, valores de IP $<1$ (peligro nulo) y valores de IP $=1-3$ (peligro bajo). De acuerdo con la Figura 3, se puede observar que los altos valores de CE, delimitan la zona del depósito de jales y algunas áreas cercanas que han sido afectadas por estos residuos, lo que sugiere que este parámetro ayuda a observar el patrón de dispersión de los residuos mineros.

En cuanto al pH, en las áreas con valores de $\mathrm{pH}$ entre 6.5 y 7.5 se localizan las muestras con valores de IP $>10$ (peligro extremo); mientras que en áreas con valores de $\mathrm{pH}$ entre 7.5 y 8 se localizan los valores de IP $=3-10$ (peligro "alto"). Finalmente, en áreas con valores de $\mathrm{pH}>8$, se localizan los valores de IP $<1$ (peligro nulo) y valores de $\mathrm{IP}=1-3$ (peligro bajo). Con esto se corrobora de forma gráfica la correlación entre el grado de contaminación y la variación del $\mathrm{pH}$ dentro de la zona de estudio.

\section{Conclusiones}

Los resultados de este estudio permiten concluir que las mediciones de la susceptibilidad magnética (SM) en los residuos de las actividades mineras y en los suelos afectados, puede ser utilizada como una medida indirecta para la identificación de estos residuos y en la delimitación de los suelos contaminados por su dispersión. Así mismo, la SM puede ser utilizada en esta zona con el fin de delimitar áreas con diferente grado de peligrosidad debido a la presencia combinada de los elementos de naturaleza tóxica como el arsénico (As), cadmio (Cd) y plomo (Pb). Además, los valores de $\mathrm{pH}$ y $\mathrm{CE}$ también pueden ser útiles en la identificación de los jales como fuente de contaminación y en la delimitación y el grado de contaminación de los suelos 


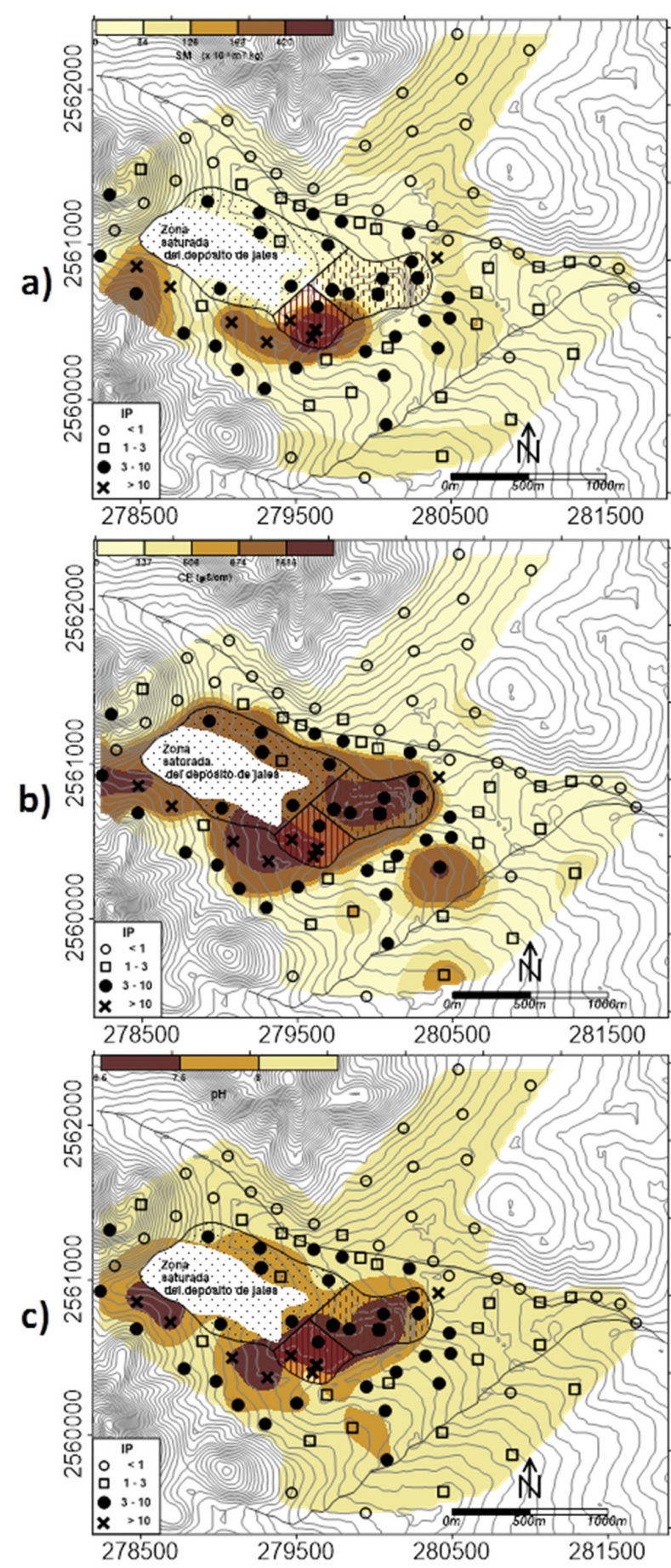

Figura 3. Mapa de distribución de los valores de IP asociados a las anomalías de SM, CE y $\mathrm{pH}$.

afectados por su dispersión.

\section{Agradecimientos}

Este estudio fue financiado por el proyecto PAPIIT IN105108. Los autores agradecen a O. Zamora, F. Vega, I. Ramos-Bautista y D. Ramos del Laboratorio de Geoquímica Ambiental del Instituto de Geología, UNAM; por su asistencia en la preparación de muestras y análisis de laboratorio. Así mismo, se agradece a T. Pi I Puig del Laboratorio de Difracción de Rayos X del Instituto de Geología y a R. I. Puente Lee del Laboratorio de Microscopía Electrónica de la Facultad de Química de la UNAM, por su colaboración en el análisis de las fases sólidas.

\section{Referencias}

American Society for Testing and Materials (ASTM), 1985, ATMS D-3987 Standard Test Method for Shake Extraction of Solid Waste with Water: Annual Book of ASTM Standards, v.11.04, 24-27 p.

Bea, S.A., Ayora, C., Carrera, J., Saaltink, M.W., Dold, B., 2010, Geochemical and environmental controls on the genesis of soluble efflorescent salts in Coastal Mine Tailings Deposits: A discussion based on reactive transport modeling: Journal of Contaminant Hydrology, 11, 65-82.

Berkowitz, B., Dror, I., Yaron, B., 2008, Contaminant geochemistry, interactions and transport in the subsurface environment: Berlin, Springer-Verlag, $412 \mathrm{p}$.

Blowes, D.W., 1997, The environmental effects of mine wastes, en Gubbins, A. (ed), Proceedings of Exploration 97: Fourth Decennial International Conference on Mineral Exploration, Mapping and Monitoring the Mine Environment, 119, 887-892.

Bowell, R. J., 1994, Sorption of arsenic by iron oxides and oxyhydroxides in soils: Applied Geochemistry, 9, 279-286.

Bradl, H., 2005, Heavy metals in the environment: Origin, interaction and remediation: Neubrucke Germany, University of Applied Sciences, Elsevier Academic Press, 282 p.

Campbell, D., Fitterman D., 2000, Geoelectrical methods for investigating mine dumps, en Proceedings of the Fifth International Conference on Acid Rock Drainage, Inc. Denver Colorado, Society for Mining, Metallurgy and Exploration, v. II, 1513-1523.

Comisión de Estudios del Territorio Nacional (CETENAL), 1978, Carta edafológica de Loreto F-14-A-71, esc. 1:50 000, México.

Comisión Nacional del Agua (CNA), 2002, Determinación de la disponibilidad de agua en el acuífero Villa de Arista, estado de San Luis Potosí: Subdirección General Técnica, Gerencia de Aguas Subterráneas, Subgerencia de Evaluación y Modelación Hidrogeológica, $20 \mathrm{p}$.

Dearing, J.A., 1994, Environmental Magnetic Susceptibility. Using the Bartington MS2 system: Kenilworth, Chi Publishing, 54 p.

Evans, M., Heller, F., 2003, Environmental Magnetism: Principles and Applications of Enviromagnetics, en Dmowska, R., Holton, J.R., Rossby, H.T. (eds), A series of monographs and textbook, The International Geophysics Series, Academic Prees Elsevier Science, $86,299 \mathrm{p}$.

Fergusson, J.E., 1990, The Heavy Elements: Chemistry environmental impact and health effects. Chemistry Department, University of Canterbury, New Zealand, Pergamon Press, 614 p.

Fialová, H., Maier, G., Petrovský, E., Kapicka, A., Boyko, T., Scholger, R., 2006, Magnetic properties of soils sites with different geological and environmental setting: Journal of Applied Geophysics. 59, 273-283.

Foster, A., Brown, E., Tingle, T., Parks, G., 1998, Quantitative arsenic speciation in mine tailings using X-ray absorption spectroscopy: American Mineralogist 83, 553-568.

Fuller, C.C., Davis, J.A., Waychunas, G.A., 1993, Surface chemistry of ferrihydrite: Part2. Kinetics of arsenate adsorption and coprecipitation: Geochimica Cosmochimica Acta 57, 2271-2282.

Goldberg, S., 1986, Chemical modeling of arsenate adsoprtion on aluminium and iron oxide minerals: Soil Science Society of American Journal, 50, 1154-1157.

Gutiérrez-Ruiz, M., Romero, F.M., González-Hernández, G., 2007, Suelos y sedimentos afectados por la dispersión de jales inactivos de sulfuros 
metálicos en la zona minera de Santa Bárbara, Chihuahua, México: Revista Mexicana de Ciencias Geológicas, 234-2, 170-184.

Holmström, H, Öhlander, B., 2001, Layers rich in Fe- and $\mathrm{Mn}$ oxyhidroxides formed at the tailings-pond water interface, a possible trap for trace metals in flooded mine tailings: Journal of Geochemical Exploration, 74, 189-203.

Johnson, R.H., Blowes, D.W., Robertson, W.D., Jambor, J.L., 1999, The hydrogeochemistry of the Nickel Rim mine tailings impoundment, Sudbury, Ontario: Journal of Contaminant Hydrology, 41(1-2), 49-80.

Levy, D.B., Custis, K.H., Casey, W.H., Rock, P.A., 1997, A comparison of metal attenuation in mine residue and overburden material from an abandoned copper mine: Applied Geochemistry, 12, 203-211.

Lin, Z., 1997, Mobilization and retention of heavy metals in mill-tailings from Garpenberg sulfide mines, Sweden: Science of the Total Environment, 198(1), 13-31.

López-Andreo, S., García-Martínez, O., Coy-Y11, R., 1987, Propiedades magnéticas de jarositas minerales y sintéticas: Boletín de la Sociedad Española de Mineralogía, 10-1, 95-96.

Lu, S., Bai, S., 2006, Study on the correlation of magnetic properties and heavy metals content in urban soils of Hangzhou City, China: Journal of Applied Geophysics, 60, 1-12.

McGregor, R., Blowes, D., Jambor, J., Robertson, W., 1998, Mobilization and attenuation of heavy metals within a nickel mine tailings impoundment near Sudbury, Ontario, Canada: Environmental Geology, 36(3-4), 305-319.

Morton-Bermea, O., Hernández, E., Soler, A., Lozano, R., González, G., Beramendi, L., Martínez, E., 2008, Utilización de herramientas sencillas para evaluar la contaminación ambiental: correlación entre propiedades magnéticas y concentración de metales pesados: Boletín de Mineralogía, 18, 69-70.

Navarro, M.C., Pérez-Sirvent, C., Martínez-Sánchez, M.J., Vidal, J., Tovar, P.J., Bech, J., 2008, Abandoned mine sites as a source of contamination by heavy metals: A case study in a semi-arid zone: Journal of Geochemical Exploration, 96, 183-193.

Ongley, L.K., Sherman, L., Armienta, A., Concilio, A., Ferguson, C., 2007, Arsenic in the soils of Zimapán, Mexico: Environmental Pollution, 145, 793-799.

Razo, I., Carrizales, L., Castro, J., Díaz-Barriga, F., Monroy, M., 2003, Arsenic and Heavy metal pollution of soil, water and sediments in a semi-arid climate mining area in Mexico: Water, Air, and Soil Pollution, 152, 129-152.

Romero, F., Armienta, M., González-Hernández, G., 2007, The solidphase control on the mobility of potentially toxic elements in an abandoned lead/zinc mine tailings impoundment, Taxco, México: Applied Geochemistry, 22, 109-127.

Romero, F., Armienta, M., Gutiérrez, M., Villaseñor, G., 2008, Factores geológicos y climáticos que determinan la peligrosidad y el impacto ambiental de jales mineros: Revista Internacional de Contaminación Ambiental, 24(2), 43-54.

Romero, F.M., Gutiérrez, M.E., 2010, Problemática ambiental asociada a jales en dos zonas mineras localizadas en el centro y sur de México: Boletín de la Sociedad Geológica Mexicana, 62(1), 43-53.
Romero, F.M., Prol-Ledesma, R.M., Canet, C., Núñez-Alvares, L., PérezVázquez, R., 2010, Acid drainage at the inactive Santa Lucia mine, western Cuba: natural attenuation of arsenic, barium and lead, and behavior of rare earth elements: Applied Geochemistry, 25, 716-727.

Selim, H.M., Sparks, D.L., 2001, Heavy metals solubility and transport in soil contaminated by mining and smelting, en Lewis (ed), Heavy metals release in soils, 89-107.

Secretaria del Medio Ambiente y Recursos Naturales (SEMARNAT), 2004, Norma Oficial Mexicana que establece los criterios para determinar las concentraciones de remediación de suelos contaminados por arsénico, bario, berilio, cadmio, cromo hexavalente, mercurio, níquel, plata, plomo, selenio, talio y/o vanadio (NOM-147SEMARNAT/SSA1): México, D.F., Diario Oficial de la Federación, 2 de Marzo de 2007, 86 p.

Spark, D., Page, A., Summer, M., Tabatabai, M., Helmke, P., 1996, Methods of soil Analysis, Part 3, Chemical Methods, en Soil Science Society of America Book Series Number 5, Madison Wisconsin, American Society of Agronomy, $1390 \mathrm{p}$.

Sultan, K., 2007, Distribution of Metals and Arsenic in Soils of Central Victoria (Creswick-Ballarat), Australia: Archives of Environmental Contamination and Toxicology, 52, 339-346.

Unites States Environmental Protection Agency (US-EPA), 1994, Method 3051: Microwave assisted acid digestion/sludges, soils (SW-846), Washington, D.C., Environmental Protection Agency Publication, $5 \mathrm{p}$.

Unites States Environmental Protection Agency (US-EPA), 1995, Method 9045C: Solid and waste $\mathrm{pH}$. Test methods for evaluating solid wastes (SW-846) Washington, D.C., Environmental Protection Agency Publication, 5 p.

Unites States Environmental Protection Agency (US-EPA), 1996a, Method 3050b: Acid digestion of sediments, sludges and soils. (SW-846), Washington, D.C., Environmental Protection Agency Publication, $12 \mathrm{p}$.

Unites States Environmental Protection Agency (US-EPA), 1996b, Method 6010b: Inductively Coupled Plasma-Atomic Emission Spectrometry (SW-846), Washington, D.C., Environmental Protection Agency Publication, $25 \mathrm{p}$.

Unites States Environmental Protection Agency (US-EPA), 2007, Method 6200: "Field portable X-ray Fluorescence Spectrometry for the determination of elemental concentrations in soil and sediment" en Test Methods for Evaluating Solid Waste, Physical/Chemical Methods (SW-846), Washington, D.C., Environmental Protection Agency Publication, $32 \mathrm{p}$.

Manuscrito recibido: Junio 7, 2013

Manuscrito corregido recibido: Agosto 31, 2013

Manuscrito aceptado: Septiembre 5, 2013 